\title{
La enfermedad como signo y las formas simbólicas del cuerpo enfermo en el horizonte de comprensión de la dermatología decimonónica'
}

\section{The disease as sign and the symbolic forms of the sick body on the horizon of comprehension $d$ the dermatology nineteenth}

\author{
Hilderman Cardona-Rodas ${ }^{a}$ \\ (D) https://orcid.org/0000-0002-6778-2102 \\ E-mail: hcardonaœudem.edu.co \\ aniversidad de Medellín. Facultad de Ciencias Sociales y \\ Humanas. Medellín, AM, Colombia.
}

\section{Resumen}

La dermatología clínica del siglo XIX fundamenta y despliega un pensamiento por similitud en una estética de la representación de lo que puede ser comprendido como enfermedad. Así, las superficies patológicas suministran un material de reflexión y apropiación del cuerpo enfermo donde confluyen cuerpo, lenguaje y acontecimiento para darle sentido a las alteraciones que confluyen en el estado patológico. Este texto reflexiona sobre esas formas simbólicas del nombrar la enfermedad que tienen lugar en descripciones, observaciones clínicas, tratados de dermatología, atlas de anatomía patológica o álbumes iconográficos del siglo XIX, donde es posible ver un régimen de visualización de lo patológico que se revela en la percepción médica de la lesión y su localización. El análisis de las enfermedades de la piel que desfiguran al rostro a partir de algunos registros clínicos e iconográficos de finales del siglo XIX pone en juego un vínculo entre imagen y palabra en términos de una eficacia dermatológica en las maneras de capturar la experiencia de la enfermedad, que tiene al cuerpo enfermo como teatro de sensibilidad performativa. Palabras clave: Cuerpo Enfermo; Dermatología Clínica; Iconografía; Lenguaje Médico; Siglo XIX.

\section{Correspondência}

Universidad de Medellín, Facultad de Ciencias Sociales y Humanas.

Carrera 87, 30-65. Medellín, AM, Colombia. CEP 050030

Este artículo se vincula con la tesis doctoral Iconografías médicas. Dermatología clínica en Colombia y España en la segunda mitad del siglo XIX, presentada en la Universitat Rovira i Virgili de Tarragona-España en 2016. 
Abstract

The clinical dermatology of the nineteenth century bases and displays a thought by similarity in an aesthetic representation of what can be understood as disease. Thus, the pathological surfaces provide a material of reflection and appropriation of the sick body where body, language and event converge to give meaning to the alterations that converge in the pathological state. This text reflects on these symbolic forms of naming the disease that occur in descriptions, clinical observations, treaties of dermatology, Atlas of pathological anatomy or iconographic albums of the nineteenth century, where it is possible to see a regime of visualization of the pathological that reveals itself in the medical perception of the lesion and its location. The analysis of skin diseases that disfigure the face from some clinical and iconographic records from the end of the 19th century puts into play a link between image and word in terms of dermatological efficacy in ways of capturing the experience of the disease, which has the sick body as a theater of performative sensitivity.

Keywords: Diseased Body; Clinical Dermatology; Iconography; Medical Language; XIX century.

\section{Introducción: Retratos de las enfermedades de la piel en el horizonte de comprensión de la dermatología decimonónica}

Los tratados, atlas, manuales o álbumes de enfermedades de la piel del siglo XIX tienen algo en común, el cuidado por un relato fiel de las perturbaciones epidérmicas en el cuerpo enfermo que revele el vínculo entre el adentro y el afuera, con una premisa clínica básica: crear una panorámica de las enfermedades más habituales y enseñar el arte de identificarlas. ${ }^{2}$

Una de las fotografías retocadas que aparece en el álbum dermatológico de Hardy y Montméja (1868), ${ }^{3}$ quienes efectúan una operación discusiva que sitúa en mutua correspondencia imagen y palabra (fotografía retocada y caracterización nosológica de las enfermedades de la piel), sobre una mujer con una lesión cancerígena en la piel, que correspondería a un caso de cancroide en los términos de Duchesne-Duparc y en la explicación dada por Hardy y Montméja, realza las implicaciones patológicas de la enfermedad sobre la frente de esta mujer (Figura 1).

El advenimiento de la fotografía, sin embargo, planteó preguntas sobre las diversas formas de representar visualmente hasta ese entonces utilizadas. Pinturas, dibujos y grabados producidos con fines científicos, y también los moldes de cera, que reproducían partes enfermas del cuerpo, comenzaron a cuestionarse en algunos de estos aspectos: fidelidad a lo real, practicidad de la realización y, principalmente, objetividad para representar. ${ }^{4}$ (Silva, 2014, p. 345, traducción propia)

\footnotetext{
Entre los tratados, atlas, manuales o álbumes que hacían parte de la pedagogía dermatológica de los médicos colombianos y españoles en el siglo XIX y comienzos del XX figuraban los siguientes (por citar solo algunos): Bateman (1818); Olive Rayer (1835); Schedel y Cazanave (1847); Ricord (1856); Bazin (1862); Duchesne-Duparc (1862); Hebra (1872, 1878); Guibout (1878, 1881a, 1881b, 1883, 1884); Besnier y Fournier (1895-1897); Hardy (1866, 1886); Hardy y de Montméja (1868); Kaposi (1881); Chatelain (1910); Brocq (1892, 1907, 1911); Brocq y Jacquet (1893); Besnier, Brocq y Jacquet (1900-1904); Fournier (1873, 1909).

3 Hardy y Montméja mencionan ensayos fotográficos británicos que motivaron la fotografía dermatológica. Dos textos destacan en este panorama, el de John Alexander Balmano Squire (1836-1908) llamado Photographs (coloured from life) of the diseases of the skin (1864 1866) y el de Georges Henry Fox (1846-1937) denominado Photographic Illustrations of Skin Diseases (1881), escritos unos años después de Hardy y Montméja, pero continúan la impronta de la fotografía dermatológica para la enseñanza de la medicina.

4 “O advento da fotografia, contudo, lançou questionamentos sobre os vários modos de representar visualmente até então existentes. Pinturas, desenhos e gravuras produzidos com fins científicos, e também os moldes em cera, que reproduziam partes doentes do corpo, todos passaram a ser questionados em algum destes aspectos: na fidelidade ao real, na praticidade de realização e, principalmente, na objetividade para representar".
} 
Figura I - Lesión cancerosa de la piel: cancroide, fotografía de la colección de l'Hôpital Saint-Louis de París, retocada con técnica colorista

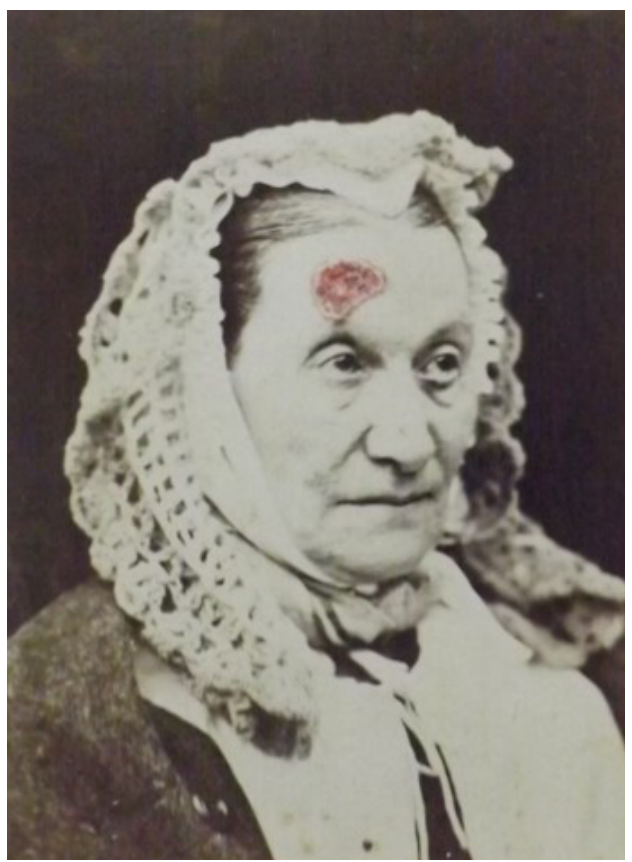

Fuente: Hardy y Montméja (1868)

El álbum de Hardy y Montméja, Clinique photographique de l'Hôpital Saint-Louis (1868), tiene la particularidad de ofrecer imágenes de dermatosis trenzadas en sus páginas en planchas fotográficas en papel albuminado.

Como alternativa al costoso sistema heliográfico, en ese momento en boga como técnica de impresión, el método de presentar fotografías originales en planchas rígidas, alternando con páginas de texto, trajo consigo la ventaja de exponer imágenes de mucha mayor calidad plástica, al tratarse de pruebas originales. ${ }^{5}$ (Silva, 2014, p. 345, traducción propia)
Estas fotografías constituían un recurso visual de utilidad didáctica para la dermatología.

La clínica como práctica discursiva se inquieta por aquello que perturba la anatomía normal del cuerpo, revelando y convirtiendo en discurso las alteraciones en la piel o en los orificios mucosos del cuerpo (boca, ano y órganos sexuales). Todo tratado de dermatología pone en escena esta inquietud. En él, el dermatólogo se sujeta a la interpretación de las superficies y revela sus riquezas: la exuberancia de la "pendiente exteriorizada" (Dagognet, 1993).

Los siglos XVII y XVIII ampliarán el estudio de las anomalías cutáneas, que eran descritas por la medicina hipocrática a partir de manifestaciones como ardor, calor, aspereza o rugosidad que alteraban la economía y se hacían visibles en la piel. Al respecto Thomas Bateman decía en su trabajo sobre enfermedades cutáneas publicado en Filadelfia en 1818:

Los nombres de los trastornos cutáneos se han utilizado en varias acepciones, y sin mucha discriminación, desde los tiempos de Hipócrates, y aún más vagamente desde el renacimiento del aprendizaje en los tiempos modernos. A partir de ese período, de hecho, las enfermedades de la piel han sido designadas generalmente por algunos pocos términos de alcance universal, que por lo tanto no portaban importación en absoluto. (Bateman, 1818, traducción propia) ${ }^{6}$

En los siglos reseñados, se constituye un ejercicio semiológico múltiple, en el que proliferan descripciones que se acompañan de nomenclaturas regidas por la percepción dermatológica. Este es el caso del médico Daniel Turner (1677-1740), quien clasificará las enfermedades cutáneas en dos grandes clases: (1) enfermedades del cráneo y del cuero cabelludo, que él denomina tiñas; y (2) las enfermedades de la

"Como alternativa ao oneroso sistema heliográfico, àquela altura em voga como técnica de impressão, o método de apresentar fotografias originais em pranchas rígidas, alternando-as com páginas de texto, trazia consigo a vantagem de expor imagens de muito maior qualidade plástica, por se tratar de provas originais".

"The names of cutaneous disorders have been used in various acceptations, and without much discrimination, from the days of Hippocrates, and still more vaguely since the revival of learning in modern times. From that period, indeed, the diseases of the skin have been generally designated by some few terms of universal import, which therefore carried no import at all". 
superficie del cuerpo, los herpes. Todo este espacio de saber conserva una filiación hipocrática-galénica, en la que se ven las diversas perturbaciones cutáneas como la expresión de un desorden humoral, el cual se expande o ejerce presión hacia fuera. Así, un examen del desbordamiento, en este ámbito de saber, permite dar cuenta del principio armónico en la organización de lo que está vivo.

La temperatura sanguínea predispone, sin ninguna duda, a las afecciones eritematosas y tuberculosas (en el sentido morfológico del término); la temperatura biliosa a las producciones pustulosas, la temperatura linfática a las enfermedades con ampollas, en fin, los individuos que presentan los atributos de temperatura nerviosa están más expuestos que los otros a las erupciones secas del liquen y del prurigo.7 (Perrin, 1988, p. 93 apud Dagognet, 1993, p. 40-41, traducción propia)

En este horizonte enunciativo, se empieza a constituir una clasificación y una distribución metódica de las innombrables enfermedades de la piel. En esta dirección, se ubican los trabajos de Jean-Louis Alibert (1768-1837), Thomas Bateman (1778-1821), Jonathan Hutchinson (1828-1913) (1888), ${ }^{8}$ Ernst Bazin (1807-1878), entre otros. ${ }^{9}$ Detengámonos en Alibert (1825), para quien la enfermedad se revela a través de criterios externos y objetivos. Para construir su árbol de las dermatosis, parte de la lesión más elemental, con el objeto de darle complejidad en un escala regularmente creciente. Lo primitivo se reconocerá por la simple inspección y palpación, ya que no presentará ningún relieve y no estará infiltrado; por el análisis, se aislará su aspecto, reduciéndolo a un casi punto, el cual,

ocupa luego la menor superficie (el puntiforme) y no tendrá ni raíz (debajo) ni ampolla (por lo alto). Esta es la mancha que le corresponde, que no varía por el color (rojo, y ella cumple el eritema o luego se vierte en la discromía). Esta estructura de base conoce también las modulaciones que no dudan en agrupar y situar. Él pasará luego a la pápula (sucedida por una ligera prominencia), después a la vesícula, porque la precedente se tiende y se hincha de serosidad, luego a la pústula (se pone a supurar), en la ampolla (toma más importancia), en fin, comienza la propia ulceración-excoriación, para terminar aun por los tubérculos, los nódulos y encanijamientos, arraigadas, algunas veces en pedículos..$^{10}$ (Dagognet, 1993, p. 43, traducción propia)

El árbol de las dermatosis que plantó Alibert tendrá una funcionalidad nosológica, en un régimen de representación de lo patológico en lo que se podría denominar una estrategia de visualización de la enfermedad. En este árbol, el tronco indica la piel; los brazos, los géneros; las ramas, las especies; y las pequeñas ramas, las variedades, escalonando y haciendo derivar las ramas secundarias, a partir de los troncos principales. El médico francés tomó prestada esta distribución del médico italiano Francisco Torti (1658-1741), quien hizo uso de este tipo de disposición genérica en su Tratado de las fiebres, publicado en 1712.

\footnotetext{
"Le tempérament sanguin prédispose, sans nul doute, aux affections érythémateuses et tuberculeuses (au sens morphologique du terme); le tempérament bilieux aux productions pustuleuses, le tempérament lympaathique aux maladies bulleuses, enfin les individus qui présentent les attributs du tempérament nerveux sont plus exposés que d'autres aux éruptions sèches du lichen et du prorrigo".

8 En el siglo XIX, en Europa, circuló la teoría del diente de Hutchinson (labio leporino), a partir de la cual el labio leporino era visto como un carácter que permitía dictaminar la presencia heredosifilítica en los diagnósticos médicos. Esta proposición tendrá un efecto de verdad en la medicina de las primeras décadas del siglo XX, en cuanto a la formación de monstruosidades y deformidades asociadas al alcohol y a la sífilis.

9 Estos médicos figuran el reportorio de personalidades que los médicos colombianos y españoles decimonónicos implementan en sus caracterizaciones clínicas de las enfermedades cutáneas”, generando así un discurso sabio en torno a la configuración de un campo de saber médico.

10 "Occupe donc le moins de surface (le punctiforme) et ne possède ni racine (en bas) ni élevure (pour le haut). C'est la macule qui lui correspond, qui ne variera que par la couleur (rouge, et elle réalise l'érythème ou alors on verse dans la dyschromie). Cette structure de base connaît bien des modulations qưon n’hésite pas à grouper et à situer. On passera ensuite à la papule (du fait d’une légère saillie), puis à la vésicule, parce que la précédente se tend et se gonfle de sérosité, puis à la pustule (elle se met à la bulle (elle prend plus d’importance), enfin on en arrive à l’ulcération-excoriation même, pour terminer encore par les tubercules, les nodules et nouures, enracinées, porfois pédiculés".
} 


\section{Figura 2 - El árbol de las fiebres de Francisco Torti, el cual tomó prestado Jean-Louis Alibert para su árbol de las dermatosis}

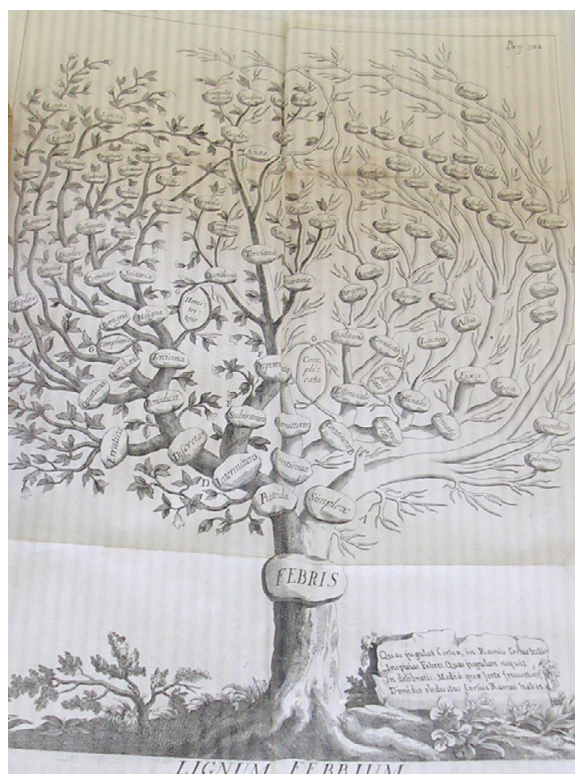

Fuente: Francisco Torti, Therapeutice specialis ad febres periodicas perniciosas (1755). Archivo Histórico del Cauca, libro 2906

Al enfatizar los criterios objetivos y puntuales, a fin de integrar los síntomas clínicos con su evolución, Alibert se libró de las distribuciones más incomprensibles y abracadabrantes; circunstancia que podría ser tomada como un punto débil en su clasificación, pero que será precisamente una fisura que permitirá la formulación de un catálogo múltiple de interpretaciones en el dominio de la mirada médica, que aprehende el hecho patológico como un conjunto de manifestaciones dermatológicas. Es así como Alfred Louis Philippe Hardy (1811-1893), en 186o, cuestionaba esta clasificación, pues el solo criterio de la lesión elemental podía confundir la percepción médica:

La lesión elemental es a menudo de poca duración; ella existe un día y, al día siguiente, no se puede constatar, sea que haya desaparecido, o bien que ella se haya modificado; a veces ella no existe. Cada vez, en esta clasificación, todas las enfermedades de hecho semejantes por su naturaleza son colocadas en clases diferentes y frecuentemente alejadas. ${ }^{11}$ (Hardy, 186o, p. 10 apud Dagognet, 1993, p. 46, traducción propia)

Las procedencias y filiaciones conceptuales para la constitución del discurso dermatológico se encuentran en las ciencias naturales (química, mineralogía y botánica), la administración, la pedagogía y la antropología propias del siglo XVIII. De este modo, dice Dagognet, una medicina especializada está determinada por las corrientes culturales poderosas de su tiempo. El campo de saber dermatológico deseará poder ordenar una realidad abundante y embrollada, privilegiando para ello un modelo tabular en el que se pone en función una taxonomía de las enfermedades según divisiones por medio de una progresión regular. Al intentar resolver el problema del nombrar, el clínico de la piel se transformará en un entomologista y en un gerente, que ve en el tegumento una fenomenología de la superficie que procura describir e individualizar, la cual "deberá saber remplazar en un conjunto eventualmente evolutivo" (Dagognet, 1993, p. 47, traducción propia). ${ }^{12}$

Dos préstamos fundamentales tienen lugar en la formación de la disciplina dermatológica: (1) al examinar el hecho primitivo o lesión elemental, lo más simple de la serie, el clínico se internará en lo basal (la mácula) sobre lo cual reposa todo el conjunto patológico, procedimiento similar al de Antoine Lavoisier (1743-1797), en química, cuando descubre las sustancias primeras, el alfabeto de la naturaleza, con sus letras y su vocabulario específico; (2) seguidamente, el clínico construye por grados de complicación una escala gradual de las enfermedades, por medio de las representaciones que se trazan con el árbol de las dermatosis (Figura 3), que ilustraba el frontispicio de la obra Clinique de l'Hôpital Saint Louis ou Traitement complet des Maladies de la Peau (1833) de Alibert, el cual constituye una arquitectura organizada en la que todos los desórdenes patológicos de la piel toman su lugar.

"La lésion élémentaire est souvent de peu de durée; elle existe un jour et, le lendemain, on ne peut plus la constater, soit qu' elle ait disparu, soit qu'elle se soit modifiée; quelquefois même, elle n’existe pas. De plus, dans cette classification, des maladies tout à fait semblables par leur nature sont rangées dans des clases différentes et souvent éloignées".

"fallait savoir replacer dans un ensamble éventuellement évolutif". 
Dos órdenes de singularidades médicas se ponen de manifiesto a través del ejercicio de la mirada dermatológica: el campo de saber de la anatomía patológica y el de la anatomoclínica.

La anatomía patológica servía para explicar las enfermedades, no para diagnosticarlas. Para la medicina anatomo-clínica, por el contrario, el diagnóstico consiste en una inferencia a partir del cuadro clínico, constatado en el viviente mediante una semiología de la vista, del tacto y del oído: hay que adivinar el estado de las partes internas del cuerpo, estado que solo puede ser directamente observado después de la muerte. El diagnóstico médico se volvió hipotético: enuncia la probabilidad de una lesión específica, pocas veces accesible a la observación. (Grmek, 1999, p. 4)

\section{Figura 3 - El árbol de las dermatosis de Jean-Louis} Alibert. óleo sobre lienzo enrollable

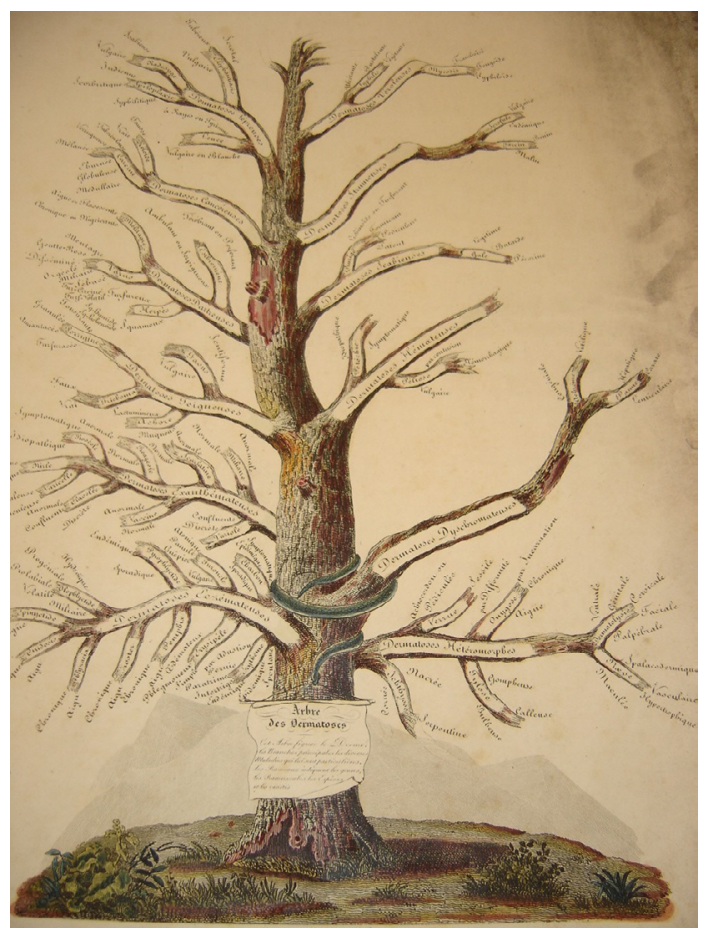

Fuente: Biblioteca Henri Feulard. Museo de l'Hôpital Saint-Louis, París
Pero será precisamente en las nosologías dermatológicas donde el aspecto externo de la enfermedad trazará un mismo registro de inteligibilidad médica, asociando síntoma y lesión. Así, se impondrá un método analítico al estudio de las enfermedades a finales del siglo XVIII y durante el XIX, método propiamente condillaciano, en el que analizar se hace en el registro de la sensación, fundamento de la experiencia y la conciencia de múltiples cadenas derivadas. ${ }^{13}$ El ejercicio perceptivo médico verá en el afuera un acontecer que deberá ser interpretado, es decir, sentido y transformado en memoria de lo corpóreo de las diversas manifestaciones de lo patológico. Esta sensación transformada construye un sentido de la realidad en el que el cuerpo es una extensión subjetiva, un operador externo, ligado a la sensación de lo que se ve, se toca, se pone frente a frente por el movimiento del lenguaje: una sensación de solidez en la autoconciencia de la mano que toca, analiza e informa. El sensualismo presupone una necesidad de aprender a sentir la corporalidad del mundo, todas sus manifestaciones (visibles en las superficies) que rodean al viviente, un mundo que es el repliegue de una realidad exterior en el sustrato de una materialidad sensible, la epidermis. He aquí las fuerzas que darán que pensar a la anatomoclínica, en lo relacionado con las experiencias de la corporalidad de lo patológico, las cuales inscriben un conocimiento de lo sensible que pasa por el ojo y el oído en la dermatología clínica decimonónica. Se intentará alcanzar un conocimiento sensorial de la enfermedad recurriendo para ello al método analítico de Étienne Bonnot de Condillac (1715-1780), consistente en "descomponer las ideas compuestas en otras simples y analizar su generación, recurriendo posteriormente al proceso de recomposición” (Arquiola, 1990, p. 215). De esta forma, una experiencia clasificatoria médica pone en juego una visibilidad de lo patológico según caracteres o hábitos exteriorizados en la perturbaciones sobre la estructura anatómica; por ello, el médico Philippe Pinel (1745-1826) afirmaba que "dada una enfermedad, determinar su verdadero

\footnotetext{
${ }^{3}$ Cf. Condillac (1963). Puede consultarse la primera versión en francés de la obra en la página de la Biblioteca Nacional de Francia: https://bit.ly/3dTkkyk.
} 
carácter, y la clase que debe ocupar en un tabla nosológica" (Arquiola, 1990, p. 217).

La aplicación del método analítico a la medicina, que dio prioridad a la exploración sensorial para establecer un diagnóstico, llevó a registrar toda singularidad patológica conforme a series de observaciones clínicas, las cuales sirvieron para educar los sentidos y establecer un lenguaje médico. Con ello, emerge un estudio de la enfermedad según el análisis de conceptos claves para la clínica: fenómeno, síntoma y signo. ${ }^{14}$ Se entenderá por fenómeno todo cambio que se produce en el cuerpo sano o enfermo, perceptible por los sentidos. Por síntoma, un cambio o alteración de las partes del cuerpo o de alguna de sus funciones, provocada por una causa morbosa, perceptible también por los sentidos. Y signo será todo fenómeno, todo síntoma, que garantiza llegar al conocimiento de los efectos más ocultos de la enfermedad. En suma, el síntoma sería el resultado de la percepción de los sentidos, mientras que el signo implica la elaboración de un juicio, de un razonamiento médico. "El proceso por el que el síntoma se convierte en signo requiere relacionar el síntoma significante con el fenómeno significado, merced a la observación fisiológica, la observación clínica y la anatomía patológica" (Arquiola, 1990, p. 226). En este orden, se crea una semiología directamente relacionada por la lesión orgánica en la que se soporta todo diagnóstico.

Con el uso del estetoscopio se afinará el sentido del oído, escuchando los sonidos corporales en los pacientes. Con ello, el método clínico de la auscultación pondrá en función la percepción de los ruidos que producen los órganos in vivo, de forma inmediata al poner la oreja cerca del pecho o mediata al emplear el estetoscopio. La intención de ver el estado anatómico, pretendió hacer externa la patología interna, haciendo visible lo invisible. Dos dispositivos, el instrumento y la visión del carácter externo, darán paso a la construcción de un conocimiento positivo de la enfermedad.

Uno de los puntos que permitió la construcción de este conocimiento positivo de la enfermedad, en la escuela anatomoclínica francesa, fue el concepto de especificidad lesional, característico del proyecto nosológico de G. L. Bayle (1774-1816). A partir de la semiología médica guiada por los signos físicos, Bayle emprendió un estudio de las lesiones orgánicas, que visualizan las lesiones en vida del enfermo, correlacionando las especies morbosas y las especies anatómicas para instaurar lo que él denominó enfermedad orgánica. Como para René Laënnec (1781-1826), Bayle verá en la enfermedad una lesión de las funciones, es decir, una lesión orgánica en cuanto un desorden de orden local o general que se manifiesta por síntomas en la alteración de las funciones orgánicas. En el proceso patogenético, la lesión vital sería la causa de la lesión orgánica, la cual produce la enfermedad y, quizá, la muerte a través de nuevas lesiones vitales. Bayle (1803 apud García Guerra, 199o, p. 246) aclara estos conceptos:

Hemos llamado lesiones vitales a todas las alteraciones de las propiedades vitales y de las funciones, y las lesiones orgánicas a todos los cambios de textura o de forma cuyas huellas se pueden reconocer después de la muerte. Las lesiones vitales pueden ser primitivas y espontáneas; no siempre determinan una degeneración orgánica, incluso cuando ocasionan la muerte. Las lesiones orgánicas, en cambio, son siempre consecutivas y no pueden producir la muerte hasta que determinan lesiones vitales. No hay, propiamente hablando, enfermedades orgánicas primitivas y espontáneas. Todas las lesiones orgánicas que se observan, sea durante la vida, sea después de la muerte, dependen de una lesión anterior de las propiedades vitales, de algún desorden en el ejercicio de las funciones, o bien son el efecto de una causa externa.

Para definir las enfermedades orgánicas recurrirá a una doble relación genética con las lesiones vitales, estableciendo una diferencia entre lesión orgánica y enfermedad orgánica:

\footnotetext{
${ }^{4}$ Se verá más adelante cómo operan estas categorías analíticas en las descripciones de diversas enfermedades cutáneas en la clínica colombiana y española en la segunda mitad del siglo XIX, guardando una directa relación con los presupuestos científicos que fundamentan la dermatología clínica europea decimonónica.
} 
Se ha dado el nombre de enfermedades orgánicas a las afecciones crónicas que dependen esencialmente de una alteración física grave y persistente de alguna parte sólida de la economía animal. Las enfermedades orgánicas son el efecto de las lesiones orgánicas. Estas últimas, como hemos dicho, están producidas por una lesión vital preexistente o por una causa externa. Pero estas enfermedades y estas lesiones no determinan padecimientos y no constituyen causas de muerte hasta que alteran a su vez las propiedades vitales, modificando o haciendo cesar el ejercicio de las funciones. (Bayle, 1803 apud García Guerra, 1990 p. 246)

Partiendo de que en la enfermedad existen lesiones orgánicas y lesiones vitales, Bayle sostiene que estas se expresan por medio de síntomas físicos o mecánicos y síntomas vitales, efectos de las enfermedades y no las enfermedades mismas, lo cual tendrá sus efectos en las caracterizaciones clínicas ligadas a un cierto sensualismo semiológico de la mirada médica.

\section{La percepción médica en las experiencias del cuerpo enfermo}

El dominio de la clínica, al emprender una caracterización y diferenciación de la enfermedad, obedecerá a un ejercicio ocular de análisis, a una soberanía de la mirada en la que el ojo sabe, decide y rige. "La clínica es probablemente el primer intento, desde el Renacimiento, de formar una ciencia únicamente sobre el campo perceptivo y una práctica sobre el ejercicio de la mirada" (Foucault, 2001, p. 130). La clínica supone una visibilidad de la enfermedad, a través de una estructura común de la mirada y la cosa vista, en la que su contraste positiviza un solo campo de saber, que devela, despliega, el secreto de la enfermedad, visibilidad que hace de la enfermedad penetrable por códigos perceptivos. Así, el signo anuncia, pronostica lo que va a ocurrir, diagnostica lo que se viene desarrollando, no da a conocer, sino que permite esbozar un reconocimiento, adelantando las dimensiones de lo oculto. En la percepción médica,

la formación del método clínico está vinculada a la emergencia de la mirada del médico en el campo de los signos y de los síntomas. El reconocimiento de sus derechos constituyentes acarrea la desaparición de su distinción absoluta y el postulado de que, en lo sucesivo, el significante (signo y síntoma) será enteramente transparente para el significado que aparece, sin ocultación ni residuo, en su realidad más maquinal, y que el ser del significado -el corazón de la enfermedad- se agotará entero en la sintaxis inteligible del significante. (Foucault, 2001, p. 132)

Más allá de los síntomas no hay esencia de lo patológico, ya que, como dice Foucault, la enfermedad es un fenómeno de sí mismo. "Una enfermedad es un todo, ya que se le pueden asignar los elementos; tiene un fin, ya que se puede calcular sus resultados; por consiguiente es un todo colocado en los límites de la invasión y de la terminación" (Broussonnet, 1797 apud Foucault, 2001, p. 133). Un síntoma tiene solo el papel de indicador soberano, es un fenómeno de una ley de aparición de la naturaleza de la enfermedad, susceptible de ser transformado en signo. De esta forma, la estructura del síntoma subyace en toda una filosofía del signo natural, en el que el pensamiento clínico no hace más que transponer una configuración conceptual fundamentada en los planteamientos de Condillac. El síntoma desempeña en la clínica el papel de lenguaje en acción según la estructura lingüística del signo. De allí que el decir enfermedad se vuelva un objeto problemático bajo los factores estructurales de la metáfora y la metonimia entre relaciones, desplazamientos y distribuciones en el campo perceptivo de la clínica, en un cuadro virtualmente ocupado por lo que puede ser visto y dicho.

Lo que el signo dice es precisamente el síntoma, su soporte morfológico. He aquí una operación en la que el síntoma se convierte en signo a través de una mirada sensible a la diferencia, a la simultaneidad, a la sucesión y a la frecuencia. "Operación espontáneamente diferencial, consagrada a la totalidad y a la memoria, calculadora también; acto que por consiguiente reúne, en un solo movimiento, el elemento y el vínculo de los elementos entre sí" (Foucault, 2001, p. 137). El análisis y la mirada clínica tienen como rasgo común sacar a la luz un orden que es el natural mismo, revelado por una "lengua bien hecha”, como decía Condillac. 
Para fijar una geometría de la visibilidad fue necesaria la puesta en obra de un lenguaje descriptivo, en que operaba un isomorfismo entre la estructura de la enfermedad y la forma verbal que la recrea. El acto descriptivo es, en este sistema de pensamiento, una percepción del ser, el cual se deja ver (a través de las manifestaciones sintomáticas) ofreciendo al dominio del lenguaje la palabra misma de la cosa que se nombra. "En la clínica ser visto y ser hablado comunican sin tropiezo en la verdad manifiesta de la enfermedad de la cual está allí precisamente todo el ser. No hay enfermedad sino en el elemento de lo visible, y por consiguiente de lo enunciable" (Foucault, 2001, p. 138). Dos registros se ponen entonces en juego en la mirada clínica: el acto perceptivo y el elemento del lenguaje.

La soberanía de la mirada también instaurará un imperio del lenguaje, en el círculo de la teatralidad del fenómeno patológico. Se ve, se devela, en las dimensiones de lo que inquieta. Los sentidos convergen en la piel, la mirada clínica regresa sobre ella, y sitúa su espacialidad en las marcas que analiza. En lo cutáneo resuenan estas huellas, allí se escenifican en una mezcla que propone un rompecabezas al diagnóstico médico, el cual intentará ensamblar los elementos (síntomas transformados en signos) en una figura inteligible en una lógica clasificatoria y analítica. El pensamiento clínico organiza un espacio de visibilidad que pretende explorar lo que tiene delante, e informa a los sentidos en una geometría que da volumen a la percepción de lo que es medido como patológico.

En este campo de experimentación visual, el registro clínico encuentra toda su eficacia enunciativa en la caracterización dermatológica de enfermedades que los médicos emprenden en la segunda mitad del XIX. En la traducción del médico colombiano Eugenio de la Hoz para la Revista de la Sociedad Médica de Bogotá No. 68, de un artículo de Eugene Guibout, publicado en L'Union Médicale de Paris en septiembre de 1881, esta preocupación designativa médica se pone aquí en función a partir del ejercicio del diagnóstico diferencial atento con el objeto de instituir un tratamiento racional para la sífilis, la escrófula y el dartro.
Las afecciones cutáneas -dice Guibout- que se revelan en una las tres diátesis, sifilítica, escrofulosa y dartrosa, presentan a veces dificultades en el diagnóstico. En efecto, todas tres tienen por terreno común la piel y se hacen notar por iguales producciones morbosas: la pápula, el tubérculo, la pústula, \&a., hay, además, en sus manifestaciones ciertas semejanzas que permiten fácilmente la confusión hasta el punto de dejar indecisos aun a los grandes maestros. Es necesario establecer de una manera exacta y precisa el diagnóstico para poder instituir un tratamiento racional. (Guibout, 1881a, p. 374)

El sitio privilegiado de la sífilis sería la frente, desde donde se diseminan una serie de pústulas denominadas corona veneris. Su presencia en la palma de las manos y de los pies (manifestada como pénfigo palmar o plantar en los recién nacidos) permitiría, dice Guibout, a diagnosticar la enfermedad. Lo contrario del dartro, que recorre todas las partes del cuerpo, bajo la forma seca, en donde la piel es dura y áspera, o bajo la forma húmeda, con la piel fina. Las formaciones de esta última enfermedad serían simétricas, es decir, "la configuración y disposición idénticas de las mismas lesiones sobre las partes correspondientes del cuerpo" (Guibout, 1881a, p. 374). Y para el caso de la escrófula, esta escoge como lugar de implantación, según el médico francés, la cara, la nariz y los pómulos.

Otro elemento no menos importante para el diagnóstico es el color. Las lesiones escrofulosas se hacen notables por un tinte rojo vino o de frambuesa, mientras que el de la sífilis es de cobre, o, si se quiere, rojo oscuro, que Mr. Hardy compara con la carne de jamón crudo. El dartro no posee color especial, es decir, que tiene todas las coloraciones. Es así que en la psoriasis es blanco y reviste tonos diferentes: rosado, plateado, mate o color de yeso. Hay otras erupciones dartrosas rojas, amarillas o de rojo vivo. (Guibout, 1881a, p. 375)

Otros de los puntos que tiene en cuenta Guibout es el estudio de las formas que toman estas tres 
diátesis. ${ }^{15}$ Compara la sífilis con un Proteo por su cambiante variabilidad morfológica.

Un enfermo, por ejemplo, que sufre de un chancro sifilítico como accidente primitivo, permanece sin tratamiento alguno, devorado por la sífilis, al fin de algunas semanas se verá aparecer en él la roséola; después de un mes, las pápulas; más tarde los tubérculos; y en el período terciario, las costras y profundas ulceraciones. (Guibout, 1881a, p. 375)

Esta misma variabilidad morfológica se hallaría en la escrófula. Al manifestarse, su color es rojo vinoso y con algunas escamas, y más tarde presenta sobre este fondo tubérculos propensos a ulcerarse. Todo lo contrario ocurre con el dartro, el cual viste siempre la misma forma durante su existencia. La primera eczema tiende a curarse, "pero el principio vicioso queda latente”, dando origen a posteriores eczemas. "El dartroso que sufre de una psoriasis será dartroso toda su vida con la misma afección" (Guibout, 1881a, p. 375).

La sífilis resulta del chancro, que es un huevo, y cuando invade la piel es, en primer lugar, bajo la forma de manchas que destruye la roséola sifilítica. Estas manchas pueden ser infinitas, diseminadas por toda la superficie del cuerpo, pero quedan siempre aisladas las unas de las otras, nunca se observan confluentes. El dartro principia por pequeños puntos que se unen y terminan por fusionarse: hay, pues, confluencia. Mientras más antigua es la diátesis, más grande es la superficie que ocupan sus manifestaciones; en la hérpetides maligna exfoliatriz no hay un solo punto sano de la piel. En la sífilis, por el contrario, la antigüedad de la diátesis hace que sus manifestaciones cutáneas se limiten y estrechen hasta agruparse en una o dos regiones del cuerpo; o ellas dejan sanas la mayor parte de la piel, y constituyen entonces las lesiones parciales descritas por Mr. Hardy con el nombre de sífilis tardías en grupos. La sífilis abandona la piel en cierta época de su evolución para atacar las vísceras imitando al dartro, que, en su curso, deja reposar la piel enferma, mientras que otros órganos internos son invadidos por el tubérculo o el cáncer. (Guibout, 1881a, p. 376)

Las destrucciones progresivas que ocasionan la sífilis y la escrófula ponen en función una operación de plegamiento entre el adentro y el afuera, en los planteamientos de Guibout, en un juego de efectos de superficie según los grados de alteración de las partes organizadas. Así, la escrófula destruye los tejidos "de la superficie o las partes profundas", mientras que la sífilis seguiría una marcha inversa, "de la profundidad a la superficie". "En ambos casos el esqueleto óseo y cartilaginoso de la nariz puede destruirse y aplanar este órgano. Si la piel ha quedado sana, diagnostíquese la sífilis; si por el contrario la ulceración de la piel procede a los huesos y cartílagos, diagnostíquese escrófula” (Guibout, 1881a, p. 376).

En estas tres diátesis, las ulceraciones tendrían un conjunto de singularidades epidérmicas con un valor de diagnóstico para el juicio médico. Estos caracteres particulares y patognomónicos por sí solos serían suficientes para establecer el diagnóstico "de la diátesis a la cual pertenecen". De esta manera, las ulceraciones de la escrófula,

[...] tienen los bordes cortados, delgados, irregulares, como festoneados y libres, de manera que pueden desprenderse con facilidad y permiten la exploración de un estilete. El fondo de la úlcera está cubierto de botones carnosos y es anfractuoso; las cicatrices que suceden son profundas, indelebles, notables por sus adherencias a los tejidos adyacentes y su aspecto reticulado; su superficie es irregular, en forma de costura por las bridas salientes que las surcan. (Guibout, 1881a, p. 377)

En la sífilis, las ulceraciones, "están hechas como por un sacabocados: su fondo es liso y de color cobre, recubierto más o menos de un pus gris" (Guibout, 1881a, p. 377). Sus cicatrices son endebles como en la escrófula, superficiales, lisas y sin adherencias a los tejidos subyacentes "sobre las cuales deslizan

Entender aquí diátesis como temperamento, constitución, hábito o predisposición individual, congénita o hereditaria a enfermar de ciertas dolencias. 
con facilidad"; "la piel se adelgaza y pierde su color que se hace menos luciente, y en su lugar existe una mancha blanquizca, punteada, análoga a la superficie de la vacuna" (Guibout, 1881a, p. 378).

Por último, las ulceraciones del dartro no afectan en profundidad, interesan tan solo la "capa más superficial de la dermis, sus bordes son irregulares, festoneados, tallados en forma de bisel, siempre adherentes y jamás libres; ellas se curan sin dejar nunca huella; no hay, pues, cicatrices" (Guibout, 1881a, p. 378).

\section{Narrativas corporales de la enfermedad en la iconografía médica}

El análisis diferencial que propone Guibout en cuanto campo de experimentación visual sobre el cuerpo enfermo tiene su correlato en la representación iconográfica. Con la publicación de fotografías y dibujos de enfermedades de la piel en los tratados y álbumes de dermatología clínica, apoyo visual para la definición y caracterización patológica, se registra la alteridad del cuerpo anormal que es codificado y vuelto comprensible por medio de una política de la exhibición, la cual intenta construir un estereotipo del otro que a su vez es una imagen inversa de sí misma y de la sociedad. Esta política de la exhibición es apreciable, como hemos visto, en la serie de fotografías médicas del Hôpital de Saint Louis en París de 1868, donde Hardy y Montméja fundaron el primer servicio fotográfico dedicado a la pedagogía clínica. Lo interesante de las fotos de este álbum radica en la forma de presentar los fotogramas: Hardy, patólogo, clasificaba las enfermedades, mientras que Montméja, jefe de oftalmología del hospital, toma las fotografías y las decora con técnica colorista para resaltar el paso de la enfermedad sobre el cuerpo retratado. Vemos dos fotogramas (Figuras 4 y 5) donde están implicados tres personajes: el paciente, el médico y el observador científico en el tejido entre la descripción médica y la emoción del sufrimiento visible. Unido a ello permite darle un sustrato enunciativo a las formas del ver y del decir la enfermedad de la piel presente en la preocupación de Guibout de garantizar un diagnóstico diferencial para "instituir un tratamiento racional" de diátesis sífilis, escrófula y dartro.
Figura ${ }_{4}$ - Síflis vegetante, fotografía de la colección de l'Hôpital Saint-Louis de París, retocada con técnica colorista

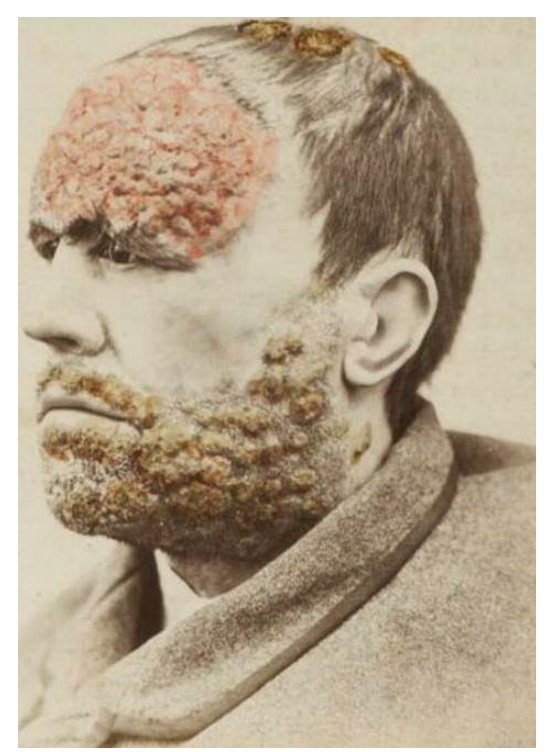

Fuente: Hardy y Montméja (1868)

Figura 5 - Scrofulide tuberculeuse, fotografía de la colección de l'Hôpital Saint-Louis de París, retocada con técnica colorista

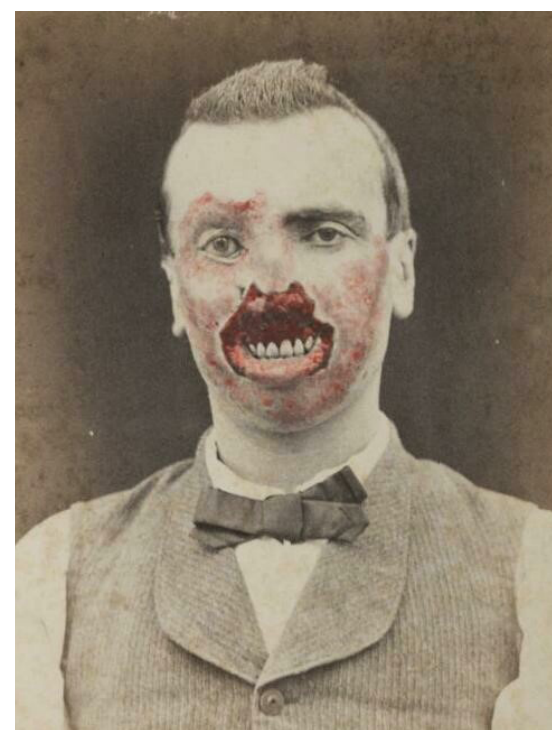

Fuente: Hardy y Montméja (1868)

Las dos reproducciones indican el carácter performativo de la enfermedad (lo cual se refleja en todos los fotogramas retocados en el álbum de estos dos médicos), donde el cuerpo enfermo 
enseña las lesiones patológicas y las huellas de la alteridad repugnante. Detengámonos un poco en la Figura 5, en la cual se ve el caso de un hombre afectado con Scrofulide tuberculeuse (lupus) en su rostro. La enfermedad es retratada como una invasión gangrenosa que descompuso su boca y fue destruyendo paulatinamente la estructura visible de su cara. Montméja resalta con un color intenso el efecto devastador del lupus, construyendo una imagen del otro que resulta a la vez atractivo y repulsivo. La fotografía exhibe igualmente, y esto para matizar el orden de lo grotesco, signos del mundo civilizado del siglo XIX en un sujeto con corbatín, chaleco y camisa impecables. Para afianzar una dialéctica entre lo sano y lo patológico, el individuo fue debidamente peinado. He aquí un cuerpo deformado que aún conserva rasgos de normalidad anatómica y moral.

Volvemos a encontrarnos con un pensamiento del afuera donde la piel es el territorio en el que se escenifica el cuerpo, el lenguaje y el acontecimiento en la pura exterioridad desplegada en el plano de la expresión y en el plano del contenido. La dermatología clínica produce sentido al hallarse con las huellas de la enfermedad sobre la piel, que la tatúan e inscriben como topografías expresivas. Un diagrama epidérmico permite entender la dinámica de estos dos planos puestos en escena por una dramática epidérmica. La expresión y el contenido se territorializan en la construcción de la enfermedad dermatológica a partir de tres posiciones intercambiables por bifurcación (Serres, 1980):

\section{Figura 6 - Diagrama epidérmico (cuerpo-lenguaje- acontecimientos)}

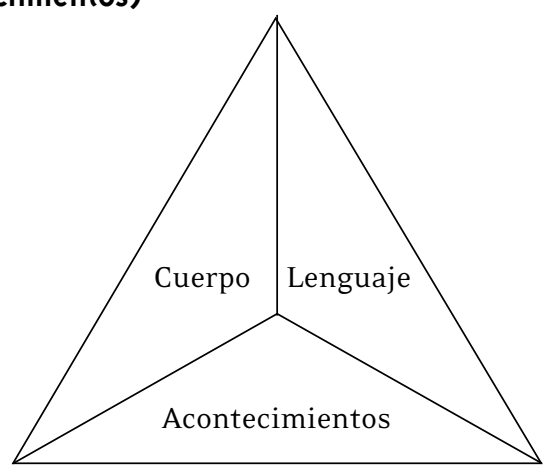

Estas tres posiciones funcionan en cuanto equivalentes. Cada una potencia a la otra en una relación de cambio y de equivalencia general. El diagrama anterior ofrece un modo de abordar el pensamiento clínico dermatológico en cuanto relación entre imagen y palabra en la segunda mitad del siglo XIX, poniendo en función un juego de relaciones de implicaciones recíprocas en las descripciones epidérmicas de enfermedades según las siguientes correspondencias:

Lenguaje-Cuerpo-Piel : Superficie-Acontecimiento-Plegamiento

Las líneas que se trazan en la dimensión del lenguaje, del cuerpo y del acontecimiento tejen un complejo conjunto de segmentaridades de los flujos entre lo visible y lo enunciable. Ráfagas de piel se vierten en la voz y en las impresiones del saber, puesto que el volumen de lo que está fuera remite a variaciones figuradas de lo que está dentro. Una operación de plegado hace ruido aquí mediante un proceso de implicación. "Si los enunciados solo existen dispersados en una forma de exterioridad, es precisamente porque las relaciones de poder son difusas, multipuntuales, en un medio que ya ni siquiera tiene forma" (Deleuze, 1987, p. 112). Así como pensar supone un ejercicio de plegado, el cuerpo, su espacialidad, dobla el exterior en un interior coextensivo a él, que funcionan como en una banda de Möebius. ${ }^{16}$ En esta relación de mutua correspondencia entre el interior y el exterior, lo patológico marcha, tiene un recorrido sintomatológico en cuanto efecto de superficie, un acontecer epidérmico, el cual traza, tatúa e imprime materialidades visibles en el cuerpo en una tensión constante entre lo que se dice y lo que se ve en el registro de la mirada clínica. La enfermedad se pone de manifiesto, como se aprecia en las descripciones clínicas dermatológicas de los médicos en la segunda mitad del siglo XIX, en una piel descubierta que confiere un territorio de visibilidad a un saber clasificatorio de la enfermedad.

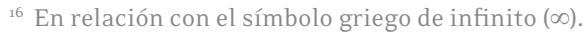


La vista de una piel enferma es sin embargo desconcertante. Los cuerpos, al exponer en superficie sus alteraciones, provocan el desvío de la mirada del otro. Los pacientes, ateridos, particularmente conscientes de las anomalías de su cuerpo, a menudo no se dejan fotografiar, sino a regañadientes. Las alteraciones se parecen, pero todas son diferentes. Al debatirse entre pústulas, máculas y pápulas, Hardy se encuentra en la obligación de recordar con vigor que la medicina no debe contentarse con asignar nombres, sino que tiene como deber contribuir a combatir el mal. De nada sirve describir, también hay que tener una idea, cuando no de las causas precisas de las patologías de la piel, al menos de la manera de aliviar el dolor. (Sicard, 1995, p. 12)

De esta forma, es posible apreciar cómo se opera una expansión del cuerpo, del lenguaje y del acontecimiento en las formas de ver y decir la enfermedad en la dimensión sensible de las superficies patológicas en el cuerpo de los pacientes. Las diversas manifestaciones patológicas suministran un material de juicio médico que permite distinguir las enfermedades a partir de análisis diferenciales, que no dejan de enfrentar interpretaciones y posiciones frente a los síntomas visibles. La enfermedad hace ruido tanto en una pluralidad de formas como en ámbitos de saber y de poder específicos. La enfermedad como lo múltiple, un barullo o una inundación de sonoridades que estremecen por sus situaciones móviles, en experiencias concretas de sensibilidad médica y de padecimientos escenificados en una pura exterioridad desplegada, que le dan materialidad y algo que pensar a la medicina.

\section{Consideraciones finales}

Solo es posible ver algo a partir de una rejilla de interpretación u horizonte posible de comprensión. De esta forma, desde el momento en que algo real es enunciado queda en la inmanencia de lo discursivamente estructurado, lo cual se refleja en las formas simbólicas del nombrar y clasificar las enfermedades de la piel, o dermatosis, en el registro de inteligibilidad de la dermatología clínica decimonónica.
El lenguaje de la piel se pone al descubierto en las superficies parlantes del cuerpo enfermo, donde confluyen el tacto y la mirada clínica. He aquí dos dimensiones del cuerpo sensible (Le Breton, 2010) que confluyen en este trabajo doctoral: el enlace entre la mirada y el tacto en la dermatología clínica conjugados en una experiencia háptica de la corporalidad de lo mórbido. Hoy en neurología se habla de una red neuronal de ojo-cerebromano que se proyecta en el cuerpo sensible; por lo que, tejiendo el argumento anterior, Richard Sennett (2009) sostendrá que la actividad táctil estimula al cerebro a pensar, que, para el caso de esta investigación, corresponde a los materiales de reflexión que suministran las huellas de la enfermedad al lenguaje de la dermatología clínica. La actividad sensorial localizada del trabajo manual deja ver el despliegue del pensamiento que se capta en la experiencia sensible del dermatólogo. Así, la dermatología clínica es el campo de la experiencia háptica del cuerpo sensible, o el dermatólogo como un artesano de la sensibilidad frente al aullido de la enfermedad en el cuerpo del paciente.

Las callosidades que se forman en las manos de quienes las utilizan profesionalmente constituyen un caso particular de tacto localizado. En principio, el engrosamiento de la piel debería insensibilizar el tacto, pero en la práctica ocurre lo contrario. Al proteger las terminaciones nerviosas de la mano, las callosidades hacen menos vacilante el acto de la exploración. Aunque todavía no se conoce bien la fisiología de este proceso, se sabe que el callo sensibiliza la mano a pequeñísimos espacios físicos y al mismo tiempo estimula la sensibilidad en las yemas de los dedos. La función del callo en la mano es comparable a la del zoom en una cámara fotográfica. (Sennett, 2009, p. 190)

La cita anterior de Sennett permite comprender el vínculo entre médico y paciente en el escenario que los conjuga: la corporalidad del hecho patológico que se hace lenguaje en las experiencias del dolor que son registradas como acontecimiento y representadas iconográficamente. Las marcas de la enfermedad, sus improntas en el cuerpo del paciente en cuanto callos que son sentidos y percibidos, tienen su pivote 
en los dibujos o fotografías que intentan darle un espacio de delimitación a lo mórbido (campo de las dermatosis), a partir de los principios de complejidad, analogía, percepción de frecuencias y cálculo del grado de certezas en el registro de la clínica dermatológica.

\section{Referencias}

ALIBERT, J. L. Description des maladies de la peau. Bruxelas: Auguste Wahlen, 1825. Disponível em: <https://bit.ly/2UFhvJL>. Acesso em: 2 abr. 2020.

ARQUIOLA, E. La aplicación del método analítico al estudio de la enfermedad en Francia en el transito del siglo XVIII al XIX. Asclepio, Madri, v. XLII, n. 1, p. 213-236, 1990.

BATEMAN, T. Practical synopsis of cutaneous diseases according to the arrangement of Dr Willan, exhibiting a concise view of the diagnostic symptoms and the method of treatment. Filadélfia: Collins \& Croft, 1818.

BAZIN, E. Leçons theoriques et cliniques sur les affections generiques de la peau. Paris: Adrien Delahaye, 1862.

BESNIER, E.; FOURNIER, J. A. Le musée de l'Hopital Saint-Louis, iconographia des malaries cutanées et syphilitiques (Atlas). Paris: Rueff et Cie, 1895-1897.

BESNIER, E., BROCQ, L.; JACQUET, L. La pratique dermatologique: traite de dermatologie appliquee. Paris: Masson et C, 1900-1904.

BROCQ, L. A. J. Traitement des maladies de la peau: avec un abrege de la simptomatologie. Paris: Octave Doin, 1892.

BROCQ, L. A. J. Traite elementaire de dermatologie pratique: comprenant les syphilides cutanees. Paris: Octave Doin, 1907.

BROCQ, L. A. J. Consultations dermatologiques. Paris: Octave Doin, 1911.

BROCQ, L.; JACQUET L. Precis elementaire de dermatologie. Paris: Masson, 1893.

CHATELAIN, E. Precis iconographique des maladies de la peau. París: A. Moloine, 1910. Con 5o planchas a color de Félix Méheux. Disponível em: <https://bit.ly/3aHs58s >. Acesso em: 2 abr. 2020.

CONDILLAC, E. B. Tratado de las sensaciones. Buenos Aires: Eudeba, 1963.

DAGOGNET, F. La peau découverte. Paris: Les Empêcheurs de Penser en Rond, 1993.

DELEUZE, G. Foucault. Barcelona: Paidós, 1987.

DUCHESNE-DUPARC, L. V. Tratado práctico de dermatosis o enfermedades de la piel clasificadas según el método natural. Madri: J. M. Ducazcal, 1862.

FOUCAULT, M. El nacimiento de clínica. Una arqueología de la mirada médica. Cidade do México: Siglo XXI, 2001.

FOURNIER, J. A. Lecons sur la syphilis: etudiee plus particulierement chez la femme. Paris: Adrien Delahaye, 1873.

FOURNIER, J. A. Traitement de la syphilis. Paris: Rueff et Cie, 1909.

FOX, G. H. Photographic Illustrations of

Skin Diseases. Nova York: E. B. Treat, 1881. Disponível em: <https://bit.ly/2UBc7qH>. Acesso em: 2 abr. 2020.

GARCÍA GUERRA, D. La lesión vital en el pensamiento nosológico de G. L. Bayle. Asclepio, Madri, v. XLII, n. 1, p. 237-253, 1990.

GRMEK, M. El concepto de enfermedad [en el siglo XIX]. In: GRMEK M. (Ed.). Historia del pensamiento médico en Occidente. Del romanticismo a la ciencia moderna. Paris: Seuil, 1999. Traducción de Jorge Márquez Valderrama.

GUIBOUT, E. Lecciones Clínicas sobre las enfermedades de la piel dadas en el Hospital de San Luis de Paris. Madri: Nicolás Mota, 1878. Tomo I.

GUIBOUT, E. Estudio comparativo de las manifestaciones cutáneas en la sífilis, la escrófula y el dartro (traducción de Eugenio de la Hoz). Revista Médica, Bogotá, v. VI, n. 68, p. 373-378, 1881a.

GUIBOUT, E. Lecciones Clínicas sobre las enfermedades de la piel dadas en el Hospital de 
San Luis de Paris. Madri: Nicolás Mota, 1881b. Tomo II.

GUIBOUT, E. Principex generaux de dermatologie; nosographie et therapeutique des maladies de la peau. Paris: G. Masson, 1883.

GUIBOUT, E. Lecciones Clínicas sobre las enfermedades de la piel dadas en el Hospital de San Luis de Paris. Madri: Nicolás Mota, 1884. Tomo III.

HARDY, A. Lecciones sobre la escrófula y las escrofúlides y sobre la sífilis explicadas en el Hospital de San Luis. Madri: Imprenta Española, 1866.

HARDY, A. Traite Pratiquet et descritiptif des maladies de la peau. Paris: J-B. Bailliere et Fils, 1886.

HARDY, A.; MONTMÉJA, A. Clinique

photographique de l'Hôpital Saint-Louis. Paris, Chamerot et Lauwereyns, 1868.

HEBRA, F. Traite des maladies de la peau. Paris:

G. Masson, 1872 .

HEBRA, F. Traite des maladies de la peau: comprenant les axenthemes aigus. Paris: G. Masson, 1878.

HUTCHINSON, J. La Sífilis. Traducción de Alfredo Opisso y Viñas. Barcelona: Ramón Molinas, 1888.

KAPOSI, M. Lecons sur les maladies de la peau. Paris: G. Masson, 1881.
LE BRETON, D. Cuerpo sensible. Santiago: Metales Pesados, 2010.

OLIVE RAYER, P. F. Traité des maladies de la peau. Paris: Chez J. B. Bailliere, 1835.

PERRIN, C. La peau ou la représentation du corps malade: Médecines antiques, médecine anatomoclinique. Nice: Université de Nice, 1988.

RICORD, P. Lettres sur la syphilis adressees a $M$. le redacteur en chef de l'Union Medicale. Paris:

Aux Bureaux de l’Union Medicale, 1856.

SCHEDEL, H. E.; CAZANAVE, A. Abrege Pratique des maladies de la peau. Paris: Labé, 1847.

SENNETT, R. El artesano. Barcelona: Anagrama, 2009.

SERRES, M. Le Parasite. Paris: Bernard Grasset, 1980. Traducción de Jorge Márquez Valderrama e Hilderman Cardona Rodas.

SICARD, M. En cuerpo y en razón. Traducción de Jorge Márquez Valderrama e Hilderman Cardona Rodas. In: PUJADE, R.; SICARD, M.; WALLACH, D. À corps et à raison, Photographies médicales 1840-1920. Paris: Marval, 1995.

SILVA, J. R. Fotografia e ciência: a utopia da imagem objetiva e seus usos nas ciências e na medicina. Boletim do Museu Paraense Emílio Goeldi. Ciências Humanas, Belém, v. 9, n. 2, p. 34336o, 2014. Disponível em: <https://bit.ly/ 2UBc7qH>. Acesso em: 2 abr. 2020. 